\title{
Genetic parameters of a random regression model for daily feed intake of performance tested French Landrace and Large White growing pigs
}

\author{
Urs SCHNYDER $^{\mathrm{a}, *}$, Andreas Hofer ${ }^{\mathrm{a}}$, \\ Florence LABROUE ${ }^{\mathrm{b}}$, Niklaus KÜNZI ${ }^{\mathrm{a}}$ \\ a Institute of Animal Science, Swiss Federal Institute of Technology (ETH), \\ 8092 Zurich, Switzerland \\ b Institut technique du porc, La Motte au Vicomte, \\ BP 3, 35651 Le Rheu Cedex, France
}

(Received 28 July 2000; accepted 21 June 2001)

\begin{abstract}
Daily feed intake data of 1279 French Landrace (FL, 1039 boars and 240 castrates) and 2417 Large White (LW, 2032 boars and 385 castrates) growing pigs were recorded with electronic feed dispensers in three French central testing stations from 1992-1994. Male (35 to $95 \mathrm{~kg}$ live body weight) or castrated (100 kg live body weight) group housed, ad libitum fed pigs were performance tested. A quadratic polynomial in days on test with fixed regressions for sex and batch, random regressions for additive genetic, pen, litter and individual permanent environmental effects was used, with two different models for the residual variance: constant in model 1 and modelled with a quadratic polynomial depending on the day on test $d_{m}$ as follows in model 2: $\sigma_{\varepsilon_{m}}^{2}=\exp \left(\gamma_{0}+\gamma_{1} d_{m}+\gamma_{2} d_{m}^{2}\right)$. Variance components were estimated from weekly means of daily feed intake by means of a Bayesian analysis using Gibbs sampling. Posterior means of (co)variances were calculated using 800000 samples from four chains (200 000 each). Heritability estimates of regression coefficients were 0.30 (FL model 1), 0.21 (FL model 2), 0.14 (LW1) and 0.14 (LW2) for the intercept, 0.04 (FL1), 0.04 (FL2), 0.11 (LW1) and 0.06 (LW2) for the linear, 0.03 (FL1), 0.04 (FL2) 0.11 (LW1) and 0.06 (LW2) for the quadratic term. Heritability estimates for weekly means of daily feed intake were the lowest in week 4 (FL1: 0.11, FL2: 0.11) and week 1 (LW1: 0.09, LW2: 0.10), and the highest in week 11 (FL1: 0.25, FL2: 0.24) and week 8 (LW1: 0.19, LW2: 0.18), respectively. Genetic eigenfunctions revealed that altering the shape of the feed intake curve by selection is difficult.
\end{abstract}

random regression / variance component / Gibbs sampling / feed intake / pig

* Correspondence and reprints

Present address: Swiss Brown Cattle Breeders' Federation, Chamerstrasse 56, 6300 Zug, Switzerland.

E-mail: urs.schnyder@braunvieh.ch 


\section{INTRODUCTION}

Today, selection of pigs for growth performance considers average daily feed intake and average daily live weight gain over the whole growing period and/or the ratio of the two, i.e. feed conversion. Average daily feed intake is negatively correlated with the leanness of the carcass. Selection for increased leanness and improved feed conversion has led to a decrease in the feed intake capacity $(F I C)$ [27]. "Modern" genotypes of pigs have a lower mean voluntary feed intake and feed intake increases at a lower rate with body weight compared to "older" genotypes [2]. In the long run, FIC might become a limiting factor for a further improvement of the efficiency of lean growth. In the past, improvement of feed conversion was mainly achieved by a reduction in the rate of fat deposition. But according to several authors, optimum levels of backfat thickness are or will soon be reached and other routes to improve feed efficiency have to be found [7,14,26]. De Vries and Kanis [5] have suggested dividing the growing period into three phases:

1. early fattening period where $F I C$ of pigs is determined by mechanical constraints and FIC is less than the optimum level of feed intake (FI(opt)), where lean deposition rate is at its maximum and fat deposition rate at its minimum for the given lean deposition rate [4],

2. intermediate fattening period where FIC is still determined by mechanical constraints but $F I C>F I($ opt $)$,

3. late fattening period where $F I C$ is determined by metabolic constraints with $F I C>F I($ opt).

Increasing FIC in period 1 to its optimum level should increase growth rate without affecting the leanness of the carcass, while increasing FIC in periods 2 or 3 would lead to fatter carcasses. Increasing FIC in period 1 while keeping $F I C$ in periods 2 and 3 constant should lead to more efficient animal growth. Webb [27] supports this view and stresses the need of further research on genetic and environmental effects on the shape of feed intake curves.

Electronic feeders installed in central testing stations allow the measurement of individual daily feed intake of performance tested growing pigs. Analyses of feed intake curves might lead to new interesting traits for pig breeders, e.g. curve parameters or feed intake capacity at different ages. One possibility to analyse feed intake curves is by means of polynomials [1] using a random regression model [22].

The objective of this study was to estimate genetic variation in feed intake curves of growing pigs and to assess possibilities to change the feed intake curve by selection. 
Table I. Number of animals with records of weekly means of feed intake per day (LW $=$ Large White; FL = French Landrace; \% = proportion of tested animals).

\begin{tabular}{lccccccccccccc}
\hline Week & 1 & 2 & 3 & 4 & 5 & 6 & 7 & 8 & 9 & 10 & 11 & 12 & 13 \\
Day & 4 & 11 & 18 & 25 & 32 & 39 & 46 & 53 & 60 & 67 & 74 & 81 & 88 \\
\hline LW & 2312 & 2263 & 2229 & 2173 & 2292 & 2255 & 2213 & 2137 & 1907 & 1227 & 509 & 131 & 14 \\
$\%$ & 95.7 & 93.6 & 92.2 & 89.9 & 94.8 & 93.3 & 91.6 & 88.4 & 78.9 & 50.8 & 21.1 & 5.4 & 0.6 \\
\hline FL & 1214 & 1192 & 1163 & 1156 & 1224 & 1183 & 1178 & 1160 & 1042 & 752 & 323 & 103 & 19 \\
$\%$ & 94.9 & 93.2 & 90.9 & 90.4 & 95.7 & 92.5 & 92.1 & 90.7 & 81.5 & 58.8 & 25.3 & 8.1 & 1.5 \\
\hline
\end{tabular}

\section{MATERIALS AND METHODS}

\subsection{Data}

1279 French Landrace (FL, 1039 boars and 240 castrates) pigs from 697 litters and 2417 Large White (LW, 2032 boars and 385 castrates) pigs from 1259 litters were performance tested in three French central testing stations from 1992-1994. For each animal tested, pedigree information of three generations of ancestors was available, which resulted in 3826 (FL) and 7784 (LW) animals in the pedigree, respectively. Growing pigs were housed in groups of 6 to 15 animals in 316 (FL) and 370 (LW) pens, respectively. Pens were equipped with one electronic feed dispenser each (Acema-48, Acemo, Pontivy, Morbihan, France), where ad libitum daily feed intake was recorded. Groups that were on test during the same period of time on the same testing station formed a batch. There was a total of 35 batches with French Landrace and 36 batches with Large White pigs. After about one week of adaptation to the automatic feed dispensers, animals were tested from $35 \mathrm{~kg}$ live body weight until they reached $95 \mathrm{~kg}$ (boars) or $100 \mathrm{~kg}$ (castrated males) live body weight, respectively. Raw data contained daily feed intake records for the whole period during which the animals were on the testing station, but records from the adaptation period were discarded. Test day one was defined as the day when animals reached $35 \mathrm{~kg}$ live body weight. Starting from there, weekly means of feed intake per day were calculated and saved as the record for the middle day of the week, in order to reduce the amount of data for the evaluations. This resulted in records for days $4,11,18, \ldots, 74,81$, and 88 (Tab. I). The last record of an animal represents feed intake of the last week before leaving the testing station after reaching $95 \mathrm{~kg}$ (entire males, candidates to selection) or $100 \mathrm{~kg}$ live body weight (castrates, slaughtered contemporaries).

The variance of an arithmetic mean of $n$ independent values is equal to the original variance of these values divided by $n$ (see e.g. [24]). Averaging daily records into weekly means therefore results in a reduction of the residual variance proportional to the number of records included in this average. Whenever 
records of more than one day per week were missing, all the records of this week were discarded and the weekly mean was set to missing, to avoid a major influence of missing records on the estimate of residual variance. Animals with less than five records of weekly means for the estimation of feed intake curves were deleted from the data set. This was also necessary if no records were available in the first three weeks of the testing period, as this might lead to poor estimates for polynomials, especially negative values for the intercept, which is not plausible.

\subsection{Model}

The following random regression model, which is a quadratic polynomial in days on test $d_{m}$ was fitted to weekly means of daily feed intake records:

$$
\begin{aligned}
y_{g h i j k m} & =\operatorname{sex}_{0 g}+\operatorname{sex}_{1 g} d_{m}+\operatorname{sex}_{2 g} d_{m}^{2} \\
& + \text { batch }_{0 h}+\text { batch }_{1 h} d_{m}+\text { batch }_{2 h} d_{m}^{2} \\
& +a_{0 i}+a_{1 i} d_{m}+a_{2 i} d_{m}^{2} \\
& +p_{0 j}+p_{1 j} d_{m}+p_{2 j} d_{m}^{2} \\
& +l_{0 k}+l_{1 k} d_{m}+l_{2 k} d_{m}^{2} \\
& +e_{0 i}+e_{1 i} d_{m}+e_{2 i} d_{m}^{2} \\
& +\varepsilon_{g h i j k m}
\end{aligned}
$$

where $\operatorname{sex}_{n g}$ and batch $_{n h}$ are fixed regressions for the gender of the animals, and the period and station of their test, respectively; $a_{n i}$ are random regressions for animal additive genetic effects; $p_{n j}, l_{n k}$ and $e_{n i}$ are random regressions for permanent environmental effects of pen, litter and the tested individual, respectively; $\varepsilon_{g h i j k m}$ is a random residual error which accounts for daily deviations of feed intake from the expected trajectory of animal $i$ on day $d_{m}$.

Model (1) can also be presented in a hierarchical form, using a quadratic polynomial as a regression function and fitting fixed (sex, batch) and random $(a, p, l, e)$ effects to regression coefficients, which can be regarded as artificial traits. What is called "permanent environmental effect of the tested individual" above, is nothing else than a residual for regression coefficients. The quadratic polynomial was chosen as a regression function for (weekly means of) daily feed intake based on results of Anderson and Pedersen [1], who showed that a cubic polynomial is sufficient to fit cumulated feed intake of growing pigs. A cubic polynomial for cumulated feed intake corresponds to a quadratic polynomial for daily feed intake, as daily feed intake can be written as the first derivative of cumulated feed intake. A higher order polynomial would fit the data better (reduce the residual variance), but would also substantially increase the number of covariances to be estimated. This additional effort does not seem to be justified, as feed intake is expected to evolve smoothly (almost linear) within the growing period considered. 
Fixed and random effects for regression coefficients were chosen based on results of Labroue [16,17], who analysed daily feed intake averaged within three growing periods (based on the same raw data) using a multivariate model. Instead of fitting a fixed effect for group size (number of pigs in a pen), a random permanent environmental effect for each pen (group of pigs housed together) was included in the model. The same fixed and random effects were applied to all three regression coefficients to guarantee a proper definition of heritability for these artificial traits (see section 2.4).

Normal distribution of feed intake data is assumed:

$$
\mathbf{y} \mid \mathbf{b}, \mathbf{a}, \mathbf{p}, \mathbf{l}, \mathbf{e}, \sigma_{\varepsilon_{m}}^{2} \sim N\left\{\mathbf{X b}+\mathbf{Z a}+\mathbf{U p}+\mathbf{V l}+\mathbf{W e}, \mathbf{I} \sigma_{\varepsilon_{m}}^{2}\right\}
$$

$\mathbf{y}$ is a vector containing feed intake data; $\mathbf{b}$ is a vector containing fixed regressions for sex and batch with a dimension three times the total number of levels of the fixed effects; $\mathbf{a}, \mathbf{p}, \mathbf{l}$ and $\mathbf{e}$ are vectors containing random regressions for additive genetic and permanent environmental effects with a dimension that is three times the number of animals in the pedigree, number of pens, number of litters and number of animals in the test, respectively; $\sigma_{\varepsilon_{m}}^{2}$ is the residual variance of day on test $d_{m}$ and $\mathbf{X}, \mathbf{Z}, \mathbf{U}, \mathbf{V}$ and $\mathbf{W}$ are incidence matrices containing regression covariables for each record.

The residuals are assumed to be independent. Two different models were applied for the residual variance. In the first model it was assumed constant over the whole testing period for all animals and in the second model all the animals were assumed to have the same residual variance on a given day on test $d_{m}$, but the course of the residual variance was modelled as follows :

$$
\sigma_{\varepsilon_{m}}^{2}=\exp \left(\gamma_{0}+\gamma_{1} d_{m}+\gamma_{2} d_{m}^{2}\right)
$$

This second model is expected to fit the data better, because the residual variance is likely to change during the testing period due to scale effects.

The following assumptions were used for the distributions of fixed and random effects (regressions):

$$
\begin{aligned}
\mathbf{b} & \sim \text { constant } \\
\mathbf{a} \mid \mathbf{A}, \mathbf{G}_{\mathbf{0}} & \sim N\left\{\mathbf{0},\left(\mathbf{A} \otimes \mathbf{G}_{\mathbf{0}}\right)\right\} \\
\mathbf{p} \mid \mathbf{P}_{\mathbf{0}} & \sim N\left\{\mathbf{0},\left(\mathbf{I} \otimes \mathbf{P}_{\mathbf{0}}\right)\right\} \\
\mathbf{l} \mid \mathbf{L}_{\mathbf{0}} & \sim N\left\{\mathbf{0},\left(\mathbf{I} \otimes \mathbf{L}_{\mathbf{0}}\right)\right\} \\
\mathbf{e} \mid \mathbf{E}_{\mathbf{0}} & \sim N\left\{\mathbf{0},\left(\mathbf{I} \otimes \mathbf{E}_{\mathbf{0}}\right)\right\}
\end{aligned}
$$

$\mathbf{A}$ is the numerator relationship matrix, $\mathbf{G}_{\mathbf{0}}$ is the (co)variance matrix of random regressions of additive genetic effects and $\mathbf{P}_{\mathbf{0}}, \mathbf{L}_{\mathbf{0}}$ and $\mathbf{E}_{\mathbf{0}}$ are (co)variance matrices for random regressions of permanent environmental effects. All these (co)variance matrices are of dimension $3 \times 3$. 
Table II. Lower diagonal elements of symmetric scale matrix $\mathbf{S}$ for inverse Wishart prior distributions of additive genetic $\left(\mathbf{G}_{\mathbf{0}}\right)$ and permanent environmental $\left(\mathbf{P}_{\mathbf{0}}, \mathbf{L}_{\mathbf{0}}, \mathbf{E}_{\mathbf{0}}\right)$ covariance matrices of random regression coefficients.

\begin{tabular}{lcccccc}
\hline Element & $\mathbf{S}(1,1)$ & $\mathbf{S}(2,1)$ & $\mathbf{S}(2,2)$ & $\mathbf{S}(3,1)$ & $\mathbf{S}(3,2)$ & $\mathbf{S}(3,3)$ \\
\hline Value & $3.075 \mathrm{e}^{-2}$ & $-4.900 \mathrm{e}^{-4}$ & $1.440 \mathrm{e}^{-5}$ & 0.0 & 0.0 & $2.500 \mathrm{e}^{-9}$ \\
\hline
\end{tabular}

Informative priors with low numbers of degrees of freedom were used for the variance components. For the $3 \times 3$ (co)variance matrices of regression coefficients $\mathbf{G}_{\mathbf{0}}, \mathbf{P}_{\mathbf{0}}, \mathbf{L}_{\mathbf{0}}$ and $\mathbf{E}_{\mathbf{0}}$, inverse Wishart distributions with five degrees of freedom were used. Prior scale matrices were equal for all four covariance matrices. Elements of scale matrices corresponding to intercept and linear regression coefficients were chosen such that their expected value corresponded to one fourth of the phenotypic (co)variances derived from Andersen and Pedersen [1]. Expected values for phenotypic (co)variances of the quadratic regression coefficient were arbitrarily set to $1.0 \mathrm{e}^{-8}$ (variance) and zero (covariances), as Andersen and Pedersen [1] included random effects for intercept, linear and quadratic regression coefficients only, when fitting a cubic polynomial in days on test for cumulated feed intake. The resulting elements of scale matrices for covariance matrices of random regression coefficients are shown in Table II. For the constant residual variance $\sigma_{\varepsilon}^{2}$ a scaled inverse Chi-square distribution with five degrees of freedom and scale parameter $s_{\varepsilon}^{2}=0.015$ was used. Priors for parameters $\gamma_{0}, \gamma_{1}$ and $\gamma_{2}$, that describe the course of the residual variance $\sigma_{\varepsilon_{m}}^{2}$ in the second model, were assumed independent of each other and normally distributed with standard deviations of $1.5\left(\gamma_{0}\right), 0.1\left(\gamma_{1}\right)$ and $0.01\left(\gamma_{2}\right)$.

\subsection{Variance component estimation}

For the estimation of (co)variance components our own programs were used, applying Bayesian methodology using Gibbs sampling [9]. The joint posterior distribution of the parameters given the data is the product of the likelihood and the prior distributions of all parameters [8]. From there, marginal distributions are derived easily, as they only have to be known up to proportionality. This results in normal distributions for fixed and random regressions and in inverse Wishart distributions for the (co)variance matrices for additive genetic and permanent environmental effects. For model 1, with a constant residual variance, the marginal distribution of $\sigma_{\varepsilon}^{2}$ is a scaled inverted Chi-square distribution. The parameters $\gamma_{0}, \gamma_{1}$ and $\gamma_{2}$, that describe the course of the residual variance $\sigma_{\varepsilon_{m}}^{2}$ in the second model, had to be sampled via a Metropolis-Hastings algorithm [12,19], as their distribution is not a standard one. In each round of Gibbs sampling, a new set of parameters $\gamma_{i}$ was sampled with a random-walk Metropolis algorithm [21]. Deviations from the current 
parameter values were generated from independent normal proposal densities with zero mean and fixed standard deviations $\left(0.04,0.002\right.$ and 0.00002 for $\gamma_{0}$, $\gamma_{1}$ and $\gamma_{2}$, respectively). The acceptance probability for this set of candidate points depends only on the ratio of the product of the likelihood and the prior densities of the parameters to be sampled, evaluated at the candidate points and the current parameter values. In each round of Gibbs sampling, this in-built Metropolis-Hastings algorithm was run until a new set of parameters $\gamma_{0}, \gamma_{1}$ and $\gamma_{2}$ was accepted.

Mixed model equations (MME) were processed block wise by means of Cholesky decomposition and backsubstitution when generating new solutions in the Gibbs sampler. For each combination of data sets (French Landrace and Large White) and models (constant and variable residual variance), four Gibbs chains were run, with 250000 samples each.

\subsection{Post-Gibbs analysis}

Burn-in for the first chain of model 1 was determined by the coupling chain method [13]. For this, a shorter chain (100000 samples) was run with different starting values for (co)variance components and fixed and random effects, but identical pseudo random number sequence. Line plots of samples of (co)variance components from every 100th round of Gibbs sampling were used to monitor convergence of the chains to identical sample values. For the other three chains of model 1 and the four chains of model 2 the same burn-in period was adopted and checked graphically on the single chains only. The coupling chain method could not be used for model 2, because in each round of Gibbs sampling the in-built Metropolis-Hastings sampler for parameters $\gamma_{0}$, $\gamma_{1}$ and $\gamma_{2}$ may cause a shift in the pseudo random number sequence relative to coupled chains. For all graphical analysis of Gibbs chains the statistical software package S-Plus [18] was used.

Effective sample size [23] of samples after burn-in was estimated for each chain using estimates of Monte Carlo variance obtained by the method of initial monotone sequence estimator [10]. This estimator was preferred by Geyer [10] over the initial positive sequence estimator, because it makes large reductions in the worst overestimates while doing little to underestimates.

Samples from the burn-in period were discarded and posterior means calculated from the remaining samples of each chain served as estimates of (co)variance components. Heritabilities and genetic and phenotypic correlations of regression coefficients were calculated from estimates of posterior means of (co)variance components as well as from samples from every 100th round of Gibbs sampling after burn-in. Density plots of calculated samples of heritabilities and correlations were made in S-Plus [18] to illustrate their distributions. 
The concept of heritability for regression coefficients is comparable to the heritability of a trait averaged over the whole testing period (e.g. average daily feed intake), it should clearly be distinguished from the heritability of a single measurement as defined in a simple repeatability model. The phenotypic covariance matrix used for calculating heritabilities and phenotypic correlations of regression coefficients is defined as the sum of additive genetic $\left(\mathbf{G}_{\mathbf{0}}\right)$ and permanent environmental $\left(\mathbf{P}_{\mathbf{0}}, \mathbf{L}_{\mathbf{0}}, \mathbf{E}_{\mathbf{0}}\right)$ covariance matrices. Residuals $\varepsilon_{g h i j k}$ (daily deviations from the fitted curve) in model (1) are expected to sum to zero within each animal, as any overall deviation from zero should be incorporated into the intercept of the fitted polynomial. The variance of these residuals depends on the length of the (time) interval which is specified rather arbitrarily (one day, one week, the entire growing period) when recording feed intake. Residuals are not part of regression coefficients and therefore the residual variance is excluded from the phenotypic covariance matrix of these artificial traits. It must be included in the definition of the phenotypic variance (and thus influence the heritability) of a single record of the trait evaluated with a random regression model, though.

For the whole testing period, additive genetic and permanent environmental variances of weekly means of daily feed intake were computed from posterior means of (co)variance components as (shown for additive genetic variance):

$$
\sigma_{G_{m}}^{2}=\phi_{\mathbf{m}}^{\prime} \mathbf{G}_{\mathbf{0}} \boldsymbol{\phi}_{\mathbf{m}}
$$

where $\sigma_{G_{m}}^{2}$ is the additive genetic variance for the day on test $d_{m}$; $\mathbf{G}_{\mathbf{0}}$ is the estimate of posterior mean for the additive genetic covariance matrix of regression coefficients and $\phi_{\mathbf{m}}^{\prime}=\left(1 d_{m} d_{m}^{2}\right)$ is a row vector containing regression covariables for the day on test $d_{m}$.

Daily variances calculated based on estimates of (co)variance matrices of additive genetic and the three permanent environmental effects as well as the residual variance were summed to get model estimates of phenotypic daily variances. These estimates of genetic and phenotypic daily variance were used to calculate heritabilities for weekly means of daily feed intake. Estimates of variances and heritability for weekly means of daily feed intake were plotted for the whole testing period.

The fit of the two models with different modelling of the residual variance was judged based on phenotypic daily variances. Model estimates calculated as shown above were compared to phenotypic daily variances calculated from data corrected for fixed effects included in the model. Two different methods were used to correct data for fixed effects. On the one hand estimates of fixed regression curves obtained with the respective models were used, and on the other hand fixed effects were estimated for each test day separately with analysis of variance function "aov" in S-Plus [18] using a fixed effect model. 


\subsection{Eigenfunctions and eigenvalues}

In order to assess the potential for genetic changes of the feed intake curve, genetic eigenfunctions and eigenvalues were calculated from additive genetic (co)variance matrices $\mathbf{G}_{\mathbf{0}}$. In order to allow for meaningful comparisons between the eigenvalues, eigenfunctions have to be adjusted to a norm of unity [15]. Therefore, estimates of genetic (co)variance matrices $\mathbf{G}_{\mathbf{0}}$ of regression coefficients were transformed into (co)variance matrices of regression coefficients based on normalised orthogonal polynomials. For this purpose normalised Legendre polynomials were used [15]:

$$
\begin{aligned}
& \mathbf{C}=\boldsymbol{\Phi} \mathbf{G}_{0} \boldsymbol{\Phi}^{\prime}=\boldsymbol{\Phi}_{1} \mathbf{K} \boldsymbol{\Phi}^{\prime}{ }_{1} \\
& \mathbf{K}=\left(\boldsymbol{\Phi}_{1}^{\prime}{ }_{1} \boldsymbol{\Phi}_{1}\right)^{-1} \boldsymbol{\Phi}_{1}^{\prime}{ }_{1} \boldsymbol{\Phi} \mathbf{G}_{\mathbf{0}} \boldsymbol{\Phi}^{\prime} \boldsymbol{\Phi}_{1}\left(\boldsymbol{\Phi}_{1}^{\prime} \boldsymbol{\Phi}_{1}\right)^{-1}
\end{aligned}
$$

$\mathbf{C}$ is a matrix containing genetic (co)variances between daily measurements of feed intake of dimension $n \times n$, where $n$ is the number of days with measurements; $\mathbf{G}_{\mathbf{0}}$ is the genetic (co)variance matrix between random regression coefficients using quadratic polynomials; $\mathbf{K}$ is the genetic (co)variance matrix between random regression coefficients using normalised second order Legendre polynomials; $\boldsymbol{\Phi}$ is a matrix of $n$ rows by three columns containing covariables for quadratic polynomials and $\boldsymbol{\Phi}_{1}$ is a matrix of $n$ rows by three columns containing covariables for normalised second order Legendre polynomials.

After transformation of $\mathbf{G}_{\mathbf{0}}$ into $\mathbf{K}$, eigenvalues and eigenvectors were calculated from $\mathbf{K}$ with S-Plus [18]. The three resulting eigenvectors were multiplied with $\boldsymbol{\Phi}_{1}$ in order to obtain the three eigenfunctions evaluated for the $n$ corresponding days with measurements. The corresponding eigenvalues indicate how much of the genetic variance of a population is explained by a given eigenfunction [15]. Therefore, eigenvalues were transformed to a percent scale, with their sum equal to $100 \%$.

\section{RESULTS AND DISCUSSION}

\subsection{Behaviour of Gibbs chains}

The coupled chains with identical pseudo random number sequence [13], to determine burn-in with model 1, resulted for both data sets in identical samples within 40000 rounds of Gibbs sampling. In order to be on the safe side for model 2, another 10000 samples were discarded.

When graphically checking whether Gibbs chains had converged to a stationary distribution within the 50000 rounds of burn-in chosen, an irregular pattern was discovered for both breeds in one of the four chains run under model 1. Especially (co)variance components of additive genetic, litter and 

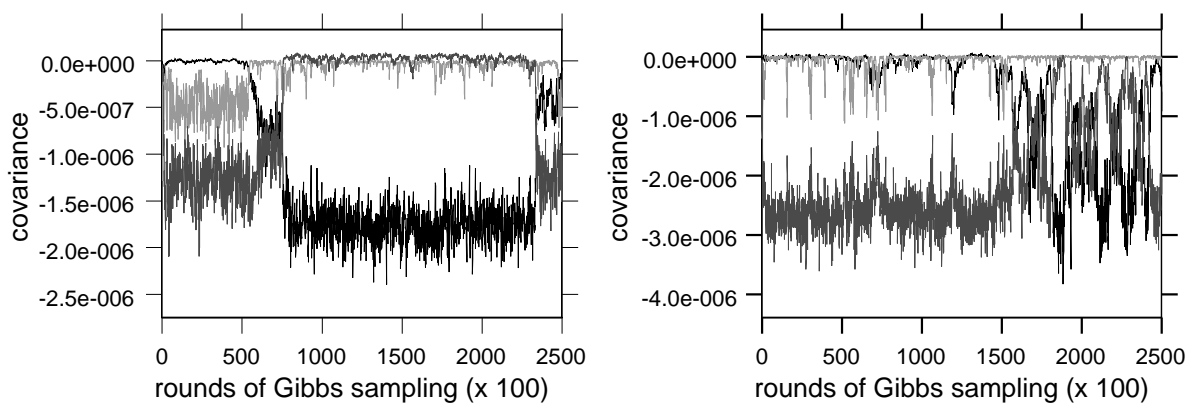

- additive genetic covariance

- litter permanent environmental covariance

— individual permanent environmental covariance

Figure 1. Gibbs samples of additive genetic, litter and individual permanent environmental covariance between linear and quadratic regression coefficients from every 100th round of the Gibbs chain with irregular behaviour under model 1 for Large White (left panel) and French Landrace (right panel) data.

individual permanent environmental effects of linear and quadratic regression coefficients were affected. This is illustrated in Figure 1 with samples of covariances between linear and quadratic regression coefficients. For Large White, the affected chain behaves "normal" for somewhat more than 50000 rounds, before the additive genetic effect absorbs most of the covariance of litter and individual permanent environmental effects. Towards the end of the chain, partition of covariance among effects is again about the same as in round 50000 (Fig. 1). Other (co)variances show a similar pattern. Only the variance of the intercept regression coefficient (for all effects) and permanent environmental effects of the pen (for all (co)variances) were not affected. For French Landrace the change in partition of (co)variances occurred after 150000 rounds, as shown in Figure 1 for the covariance between linear and quadratic regression coefficients. For the remaining rounds, fluctuations of samples were rather large compared to earlier rounds and not as stable as in the affected period of the Large White chain. For French Landrace, the variance of the intercept regression coefficient was also affected, but no changes in pen and litter (co)variances were found. For both breeds none of the other Gibbs chains showed a similar pattern, neither the three other chains run with model 1, nor the four chains run with model 2. For these chains a burnin period of 50000 rounds of Gibbs sampling seems to be sufficient by far. They seem to have reached their stationary distribution already after a few thousand rounds. The reasons for this strange behaviour discovered in two Gibbs chains are not entirely clear. With the proper prior distributions chosen for random effects and (co)variance components, property of the posterior 
distribution should be guaranteed. Gibbs sampling programs were carefully checked for errors, and were found to work correctly. Pseudo random number sequences used were different for the affected chains of the two breeds, and showed no problems when used for the other model-breed combinations. We therefore believe that the Gibbs sampler reached this different configuration of (co)variance distribution among additive genetic and permanent environmental effects for regression coefficients in the affected chains just by chance. This configuration may be supported by the data with some low probability, but is not likely to represent the true state of nature. Slow mixing of Gibbs chains may be the reason why the sampler got stuck in this configuration for so many rounds of Gibbs sampling. Because samples of (co)variances left what is believed to be the true highest density region of the stationary distribution for a substantial number of rounds, we decided not to use the affected chains for inferences on model parameters. Increase in additive genetic and decrease in permanent environmental (co)variance of regression coefficients would have had a major impact on estimates of heritabilities. To guarantee a fair comparison of results between the two models, one additional Gibbs chain was run for both breeds with model 1 , which behaved completely normal for both breeds. Thus, inferences on model parameters are based on four chains with a total of 800000 samples (after burn-in) for all four model-breed combinations.

Sums of estimates of effective sample size of the four chains run for each model-breed combination are shown in Tables III (Large White) and IV (French Landrace). For all model-breed combinations, the lowest estimates of effective sample size were found for estimates of additive genetic (co)variance components. Low estimates of effective sample size indicate slow mixing of Gibbs chains, which is considered the main reason for the long burn-in period that was chosen. Within effects, the estimates are the lowest for variances of linear and quadratic regression coefficients and their covariance, with the exception of permanent environmental effect of pens for Large White (both models) and individual permanent environmental effects under model 2 for both breeds. Highest estimates of effective sample size were found for parameters describing the residual variance and for (co)variances of permanent environmental effects of pens. On average, permanent environmental effects of pens were estimated based on records of 6.5 animals for Large White and 4.1 animals for French Landrace, respectively. For all other random effects of regression coefficients the average number of animals with records per level of effect is much lower. The number of animals with records was 1.9 per litter for Large White and 1.8 per litter for French Landrace, respectively, one per level of individual permanent environmental effect and considerably less than one per level of additive genetic effect ( 0.31 for Large White and 0.33 for French Landrace, respectively, including ancestors in the pedigree). Mixing of Gibbs chains for (co)variance components of random regression coefficients thus seems to 
Table III. Sums of estimates of effective sample size for elements of covariance matrices of intercept, linear and quadratic regression coefficients for daily feed intake (both models), the constant residual variance (model 1) and parameters $\gamma_{i}$ describing the course of the residual variance under model 2, based on samples after burn-in of four Gibbs chains (800000 samples total). Large White data.

\begin{tabular}{llcccccc}
\hline Model & Effect/element & $(1,1)$ & $(2,1)$ & $(2,2)$ & $(3,1)$ & $(3,2)$ & $(3,3)$ \\
\hline \multirow{4}{*}{ Model 1 } & Additive genetic & 250 & 265 & 58 & 241 & 52 & 52 \\
& Perm. env. pen & 20451 & 18198 & 20113 & 18523 & 21703 & 23717 \\
& Perm. env. litter & 1983 & 254 & 90 & 243 & 88 & 87 \\
& Ind. perm. env. & 800 & 692 & 493 & 594 & 431 & 396 \\
& Residual variance & 199702 & & & & & \\
\hline \multirow{5}{*}{ Model 2 } & Additive genetic & 216 & 128 & 51 & 106 & 53 & 61 \\
& Perm. env. pen & 20102 & 19180 & 21121 & 20277 & 22475 & 25498 \\
& Perm. env. litter & 1527 & 282 & 105 & 291 & 109 & 115 \\
& Ind. perm. env. & 505 & 329 & 484 & 253 & 443 & 414 \\
& $\gamma_{0}, \gamma_{1}, \gamma_{2}$ & 38025 & 33130 & 34576 & & & \\
\hline
\end{tabular}

Table IV. Sums of estimates of effective sample size for elements of covariance matrices of intercept, linear and quadratic regression coefficients for daily feed intake (both models), the constant residual variance (model 1) and parameters $\gamma_{i}$ describing the course of the residual variance under model 2, based on samples after burn-in of four Gibbs chains (800000 samples total). French Landrace data.

\begin{tabular}{llcccccc}
\hline Model & Effect/element & $(1,1)$ & $(2,1)$ & $(2,2)$ & $(3,1)$ & $(3,2)$ & $(3,3)$ \\
\hline \multirow{4}{*}{ Model 1 } & Additive genetic & 635 & 259 & 196 & 222 & 205 & 245 \\
& Perm. env. pen & 18064 & 9874 & 2910 & 8846 & 2651 & 2781 \\
& Perm. env. litter & 3506 & 1147 & 546 & 931 & 529 & 554 \\
& Ind. perm. env. & 1028 & 996 & 1260 & 1071 & 1394 & 1446 \\
& Residual variance & 189164 & & & & & \\
\hline \multirow{5}{*}{ Model 2 } & Additive genetic & 636 & 275 & 140 & 267 & 199 & 207 \\
& Perm. env. pen & 17138 & 10380 & 2656 & 9568 & 2414 & 2523 \\
& Perm. env. litter & 3558 & 1683 & 358 & 1507 & 347 & 366 \\
& Ind. perm. env. & 1235 & 1370 & 850 & 1162 & 764 & 715 \\
& $\gamma_{0}, \gamma_{1}, \gamma_{2}$ & 24512 & 10598 & 10954 & & & \\
\hline
\end{tabular}

depend on the amount of information available in the data to estimate each level of the random effect considered. For most parameters, estimates of effective sample size are not high enough to allow for accurate density estimates. For this purpose at least a few thousand independent samples from the posterior distribution are required [20]. Therefore only estimates of posterior means will 
Table V. Heritabilities (bold), genetic (above diagonals) and phenotypic (below diagonals) correlations of intercept, linear and quadratic regression coefficients for daily feed intake.

\begin{tabular}{llrrrrrr}
\hline Model $\backslash$ Breed & \multicolumn{3}{c}{ Large White } & \multicolumn{3}{c}{ French Landrace } \\
\hline \multirow{3}{*}{ Model 1 } & Intercept & $\mathbf{0 . 1 4}$ & 0.01 & 0.02 & $\mathbf{0 . 3 0}$ & -0.62 & 0.36 \\
& Linear & -0.47 & $\mathbf{0 . 1 1}$ & -0.84 & -0.48 & $\mathbf{0 . 0 4}$ & -0.27 \\
& Quadratic & 0.29 & -0.89 & $\mathbf{0 . 1 1}$ & 0.31 & -0.91 & $\mathbf{0 . 0 3}$ \\
\hline \multirow{3}{*}{ Model 2 } & Intercept & $\mathbf{0 . 1 4}$ & -0.04 & 0.10 & $\mathbf{0 . 2 1}$ & -0.51 & 0.26 \\
& Linear & -0.52 & $\mathbf{0 . 0 6}$ & -0.73 & -0.53 & $\mathbf{0 . 0 4}$ & -0.36 \\
& Quadratic & 0.33 & -0.89 & $\mathbf{0 . 0 6}$ & 0.36 & -0.92 & $\mathbf{0 . 0 4}$ \\
\hline
\end{tabular}

be given and density plots of every 100th sample can only give an indication of distributions.

\subsection{Heritabilities and correlations}

Estimates of heritabilities and correlations of regression coefficients for daily feed intake are shown in Table V. French Landrace pigs showed a quite high heritability for the scalar regression coefficient with model 1 , which was reduced substantially under model 2 , but still remained higher than for Large White pigs. Heritabilities for linear and quadratic regression coefficients were higher for Large White pigs than for French Landrace, but also reduced under model 2 compared to model 1 (Tab. V). These heritabilities already show that it is easier to change the overall level (associated with the intercept regression coefficient) than the shape of feed intake curves (associated with linear and quadratic regression coefficients).

Phenotypic correlations were very similar for both breeds and also between models, whereas genetic correlations differed substantially between breeds (Tab. V). For French Landrace, genetic correlations between the intercept and linear as well as quadratic regression coefficients were more in line with phenotypic correlations than for Large White. Differences between genetic and phenotypic correlations between linear and quadratic regression coefficients were smaller in Large White than in French Landrace. The reason for these differences may be found in (co)variance components of individual permanent environmental regression coefficients, as pen and litter explain only a small part of permanent environmental variation (data not shown).

Eissen [6] estimated heritabilities and correlations of feed intake curve parameters in a two step approach. First he fitted linear polynomials depending on days on test to daily feed intake records of growing Duroc pigs. Afterwards, he used an intercept, linear regression coefficient and residual standard deviation of the fit for individual pigs in a multivariate analysis. For both intercept and linear 
regression coefficient, he found a heritability estimate of 0.32 , which, except for the intercept of French Landrace, is much higher than our estimates for the corresponding parameters (Tab. V). His estimates of genetic and phenotypic correlations between the intercept and linear regression coefficient are -0.38 and -0.62 , which are in the same range as our estimates, except for the genetic correlations of Large Whites (Tab. V).

Density plots (Figs. 2 and 3) of heritabilities and correlations calculated from every 100th sample of (co)variances indicate how accurate these parameters can be estimated from our data. Phenotypic correlations were estimated very well for all model-breed combinations, as can be seen from their high and narrow density plots. On the contrary, density plots of genetic correlations (note the different scales) are flat over almost the whole parameter space. This indicates, that information on genetic parameters is very limited in both data sets, which may also be the reason for the slow mixing of genetic parameters. Differences between models in the shape of density plots of correlations are small and must be interpreted carefully, as estimates of effective sample size were very low for additive genetic (co)variances (Tabs. III and IV). A little difference can be found for the genetic correlation between linear and quadratic regression coefficients in Large Whites (Fig. 2), which shows a little peak close to the lower end of the parameter space in model 1 and not in model 2 . For phenotypic correlations only positions of means differ slightly. Density plots of heritabilities are intermediate in height and width compared to genetic and phenotypic correlations. Heritabilities show more accentuated peaks for linear and quadratic regression coefficients than for the intercept regression coefficient. This may be due to the fact that these low heritabilities are situated close to the lower limit of the parameter space.

\subsection{Course of variances and heritabilities}

Figures 4 and 5 show the course of the additive genetic variance, the sum of the three permanent environmental variances and the residual variance for weekly means of daily feed intake estimated with models 1 and 2 for Large White and French Landrace growing pigs. As litter and pen explain only a very small part of the total variation, permanent environmental variances were summed to reduce the number of lines in the figures. Variances were plotted for the first eleven weeks on test only, as there are substantially fewer animals with records in weeks 12 and 13 (Tab. I). The course of variances was quite similar for both models, except for the residual variance, which was constant in model 1, while it started low in model 2 and got quite high towards the end of the testing period. For both breeds, the sum of permanent environmental variances for model 1 was smaller in the beginning and larger towards the end of the testing period than for model 2. Under model 2, lower residual variance in early test weeks was partly compensated by higher permanent 

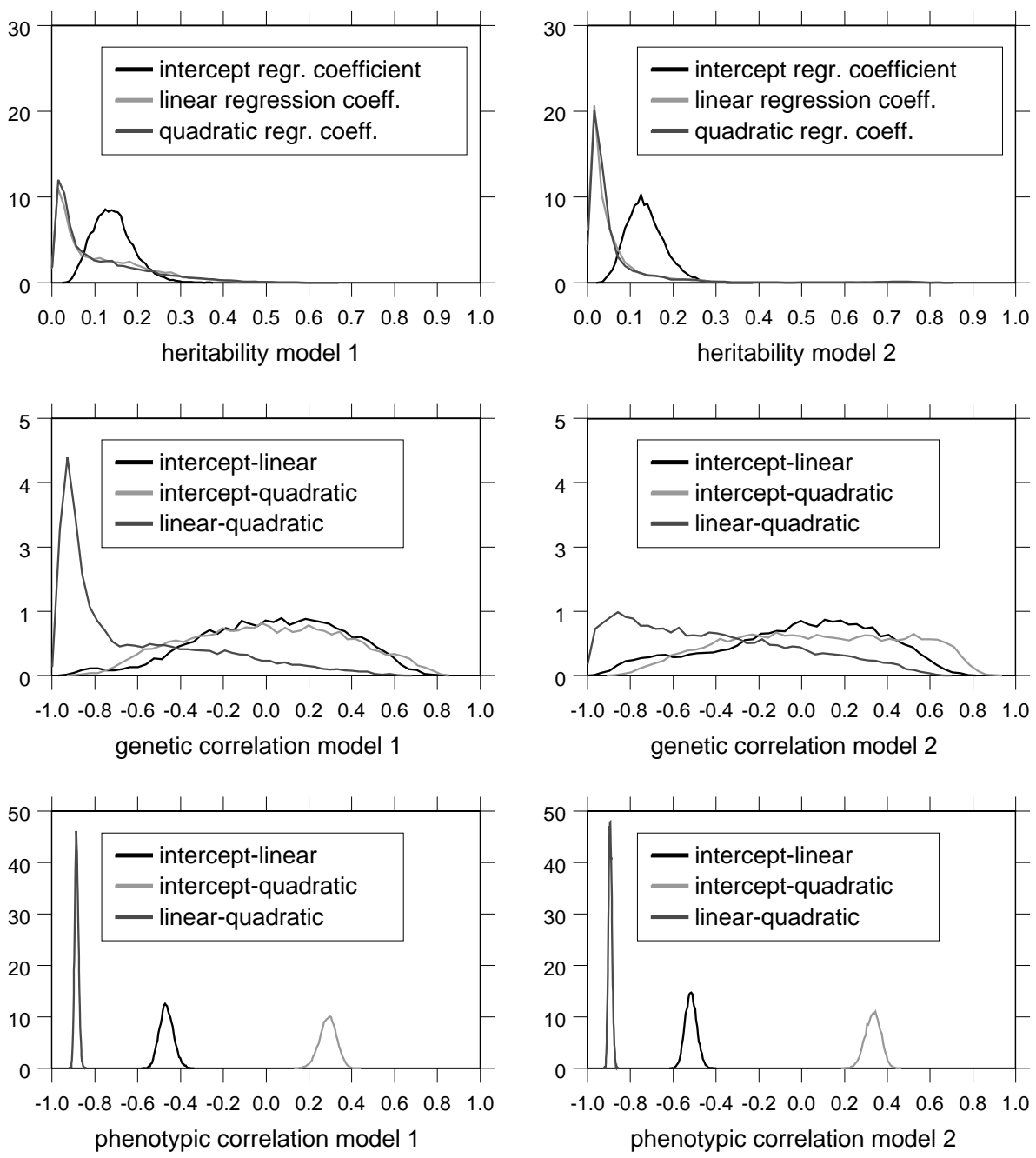

Figure 2. Density plots of heritabilities, genetic and phenotypic correlations of intercept, linear and quadratic regression coefficients for Large White, calculated from every 100th Gibbs sample of covariance matrices used for inferences under model 1 and model 2 (8 000 samples each).

environmental variance, and vice versa for late test weeks. While the course of genetic variance was similar for Large White and French Landrace pigs, the pattern shown for the permanent environmental variance was different and less regular than for the genetic variance. This also had an influence on the course of heritabilities for weekly means of feed intake per day (Fig. 6), which showed a different pattern for French Landrace than for Large White pigs. The general rise of variance during the testing period may partly be due to the 

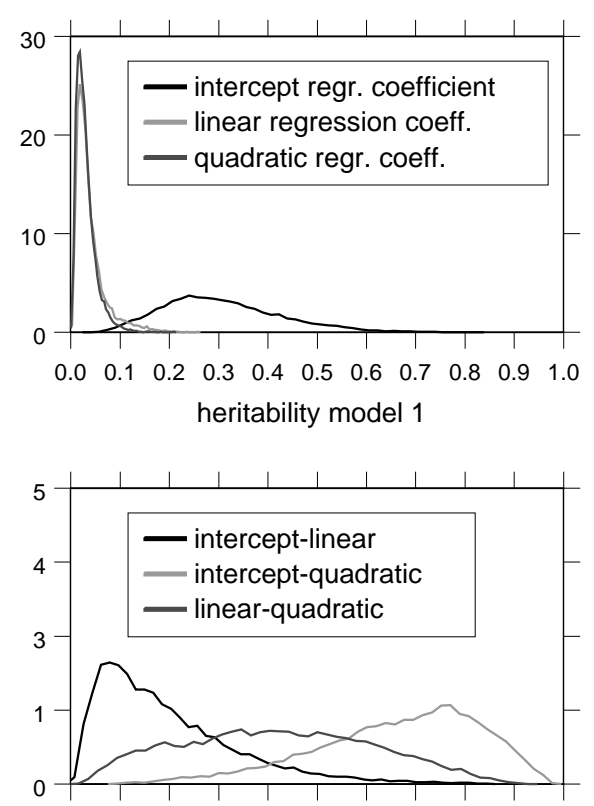

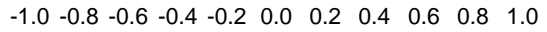

genetic correlation model 1

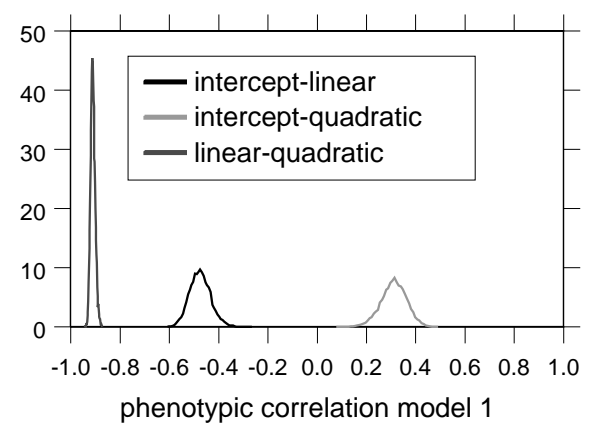

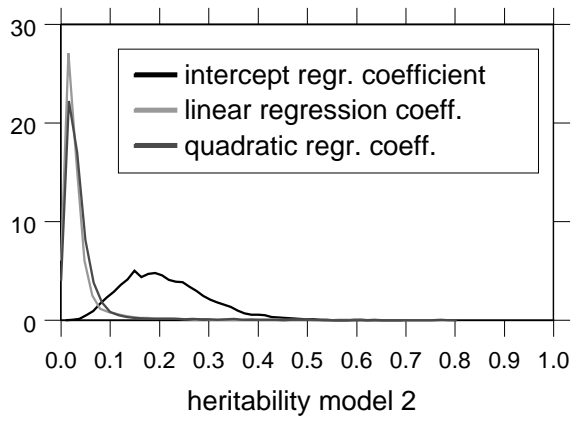
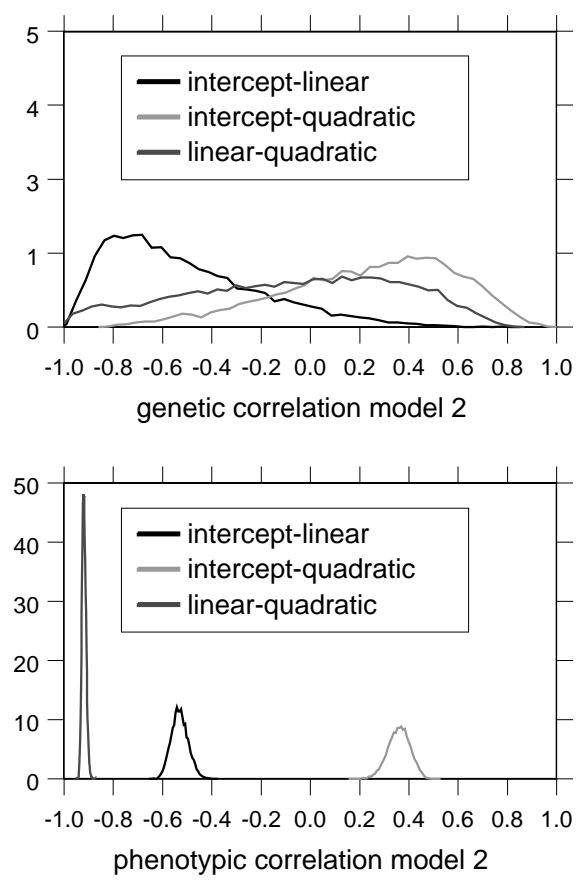

Figure 3. Density plots of heritabilities, genetic and phenotypic correlations of intercept, linear and quadratic regression coefficients for French Landrace, calculated from every 100th Gibbs sample of covariance matrices used for inferences under model 1 and model 2 (8 000 samples each).

fact that the feed intake capacity of animals increases with age and size, but it may also be influenced by variable length of testing periods, as less (slower growing) animals have records in the last two or three weeks (Tab. I). Because length of testing periods of individual pigs depends on body weight gain, fit of polynomials for faster growing pigs is based on less records than for the slower growing pigs. As accuracy of polynomial fit can only be guaranteed between the first and last record of an individual pig, daily variance may be 


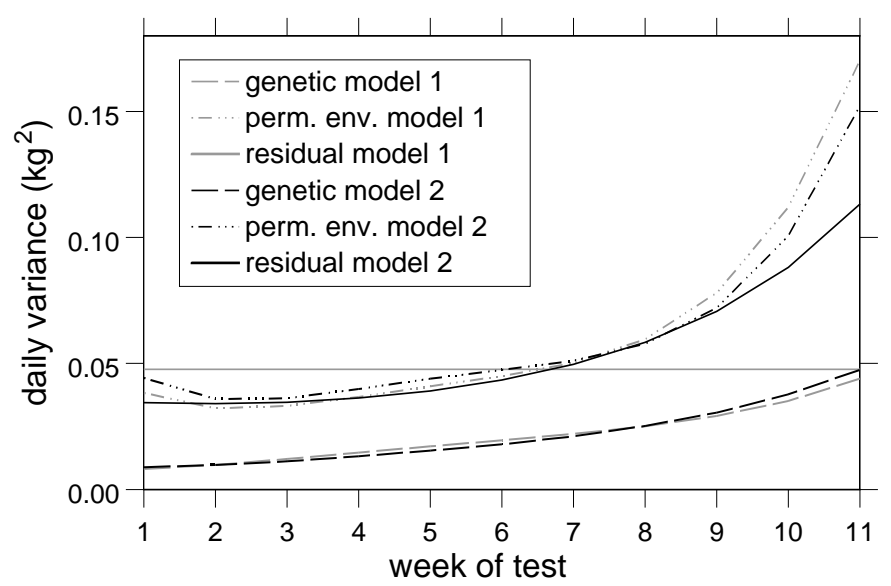

Figure 4. Course of variances for daily feed intake (kg) of Large White growing pigs for models 1 and 2.

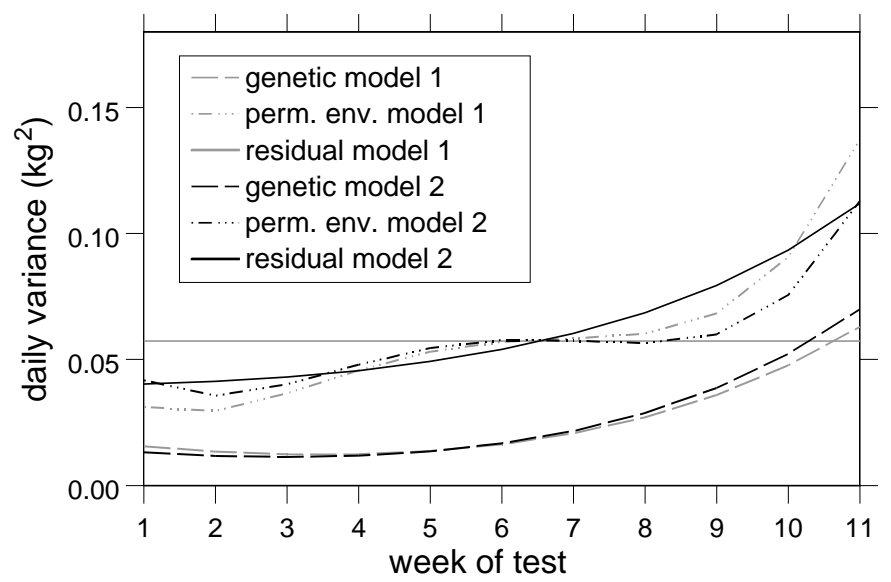

Figure 5. Course of variances for daily feed intake $(\mathrm{kg})$ of French Landrace growing pigs for models 1 and 2.

overestimated for late weeks because polynomials of fast growing pigs are not accurate any more.

The heritabilities found in this study were substantially lower than the estimate of 0.42 for average daily feed intake found by Labroue et al. [17] for the same data. Most of this difference may be explained by the difference in methodology, as the residual variance (around curves) is reduced by averaging daily feed intake over the whole testing period. Compared to the model with weekly means of daily feed intake that was used here, the residual variance is reduced by a factor equal to the average number of weeks that animals were on test. In her Ph.D. Thesis, Labroue [16] also estimated heritabilities for weekly 


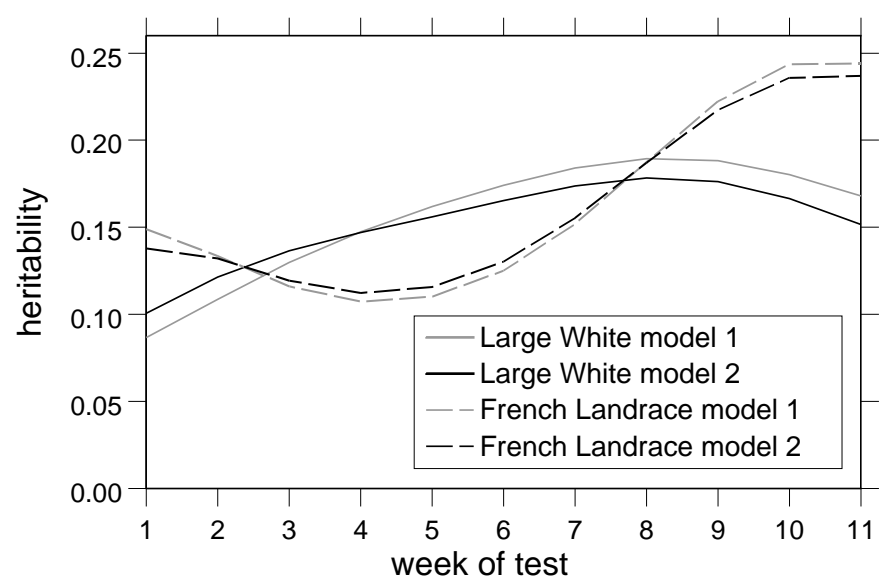

Figure 6. Course of heritabilities for daily feed intake (kg) of Large White and French Landrace growing pigs for models 1 and 2.

means of feed intake in weeks 2, 6 and 10 of the testing period based on the same data. These estimates are lower than the estimate for average daily feed intake, but on average still 0.1 higher than our results (Fig. 6), except for the slightly lower estimate of heritability in week 6 for the French Landrace. These differences cannot be explained by reduction of residual variance, as weekly means of daily feed intake were used in both studies. One possible reason is the differences in effects included in the models. Labroue [16] used a fixed effect for group size, while group effects were included as a random permanent environmental effect of the pen in this study. But as variance of permanent environmental effect of pens is small compared to additive genetic variance, this explains only about ten percent of the differences in heritability estimates.

Our heritability estimates for weekly means of daily feed intake are slightly lower than the values found by Von Felde et al. [25]. Heritability estimates of Hall et al. [11] for four biweekly means of daily feed intake lay in between the ones found here and those of Von Felde et al. [25]. They are comparable to our results for Large White pigs, if the reduction of the residual variance due to biweekly means (compared to weekly means) is accounted for. The estimate of de Haer and de Vries [3] for heritability of average daily feed intake lies in the middle range of our estimates for weekly means of daily feed intake, while estimates from other studies are higher $[6,11,17,25]$.

\subsection{Model fit}

Estimates of feed intake curves for fixed effects of sex (Fig. 7) were almost identical for both models. For males, fit of polynomials with estimates from single test weeks was very good for the first eight test weeks, while differences 


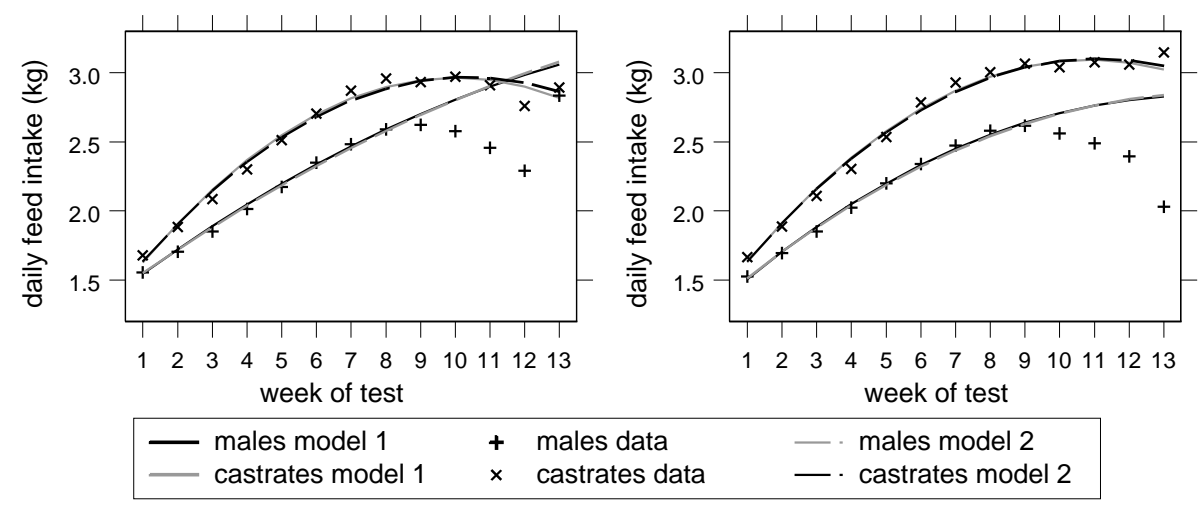

Figure 7. Course of weekly means of daily feed intake $(\mathrm{kg})$ for males and castrates of Large White (left panel) and French Landrace (right panel) growing pigs estimated with models 1 and 2, as well as from data of each test week separately.

got more pronounced as the number of animals with records decreased. For castrates, fit of polynomials was better in late test weeks than for males. As castrates grow slower on average than males, a higher proportion of castrates had records in late test weeks, which led to a better fit of polynomials in late test weeks. Phenotypic variances of weekly means of daily feed intake (Fig. 8) were very similar for both methods of correcting data for fixed effects. Therefore only results of the analysis of variance are shown, using a fixed effects model for each test week separately. As expected from the model, estimates from model 2 were closer to estimates from data corrected for fixed effects (sex and batch) for the first eight weeks of the testing period than estimates from model 1. For the remaining five weeks, estimates from model 1 are better, except for the last week of the French Landrace. Differences between breeds in phenotypic variance estimated from corrected data for last test weeks occurred only by chance, as a few castrated French Landrace pigs with big differences in weekly means of daily feed intake happened to be paired in two batches. Generally, model estimates of phenotypic variance were too high for later test weeks, where the number of animals with records was reduced (Tab. I). This supports that polynomials fitted to feed intake records of fast growing pigs may be inaccurate after they finished the test and therefore cause overestimated daily variances for late weeks (see Sect. 3.3 and Figs. 4 and 5).

For both breeds, curves of residual variances of models 1 and 2 intersect between weeks 6 and 7 (Figs. 4 and 5). The constant residual variance in model 1 is likely to overestimate the true residual variance in the first and to underestimate it in the second half of the testing period. The quadratic polynomial used to fit the natural logarithm of the residual variance of each test day in model 2 (equation (3)), results in an almost perfect fit of phenotypic test 

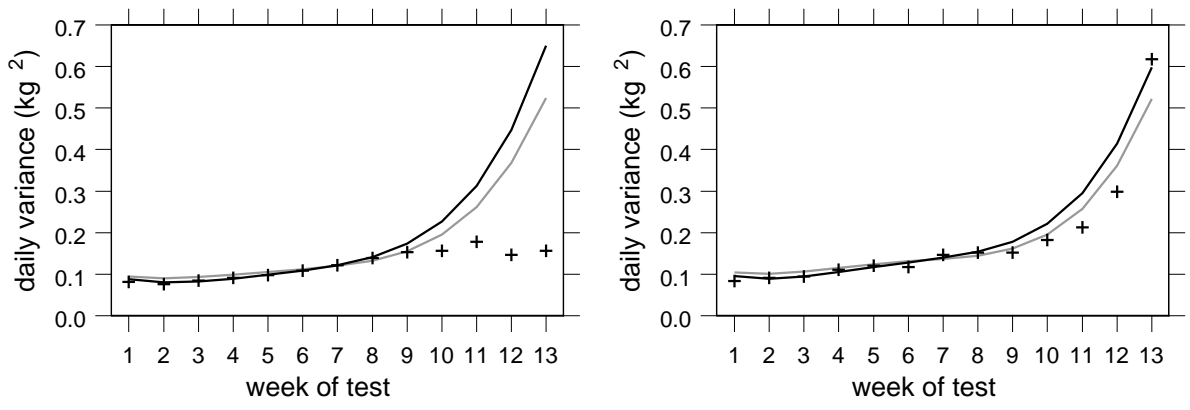

model 1

$+\quad$ corrected data

model 2

Figure 8. Course of phenotypic variance for weekly means of daily feed intake $(\mathrm{kg})$ of Large White (left panel) and French Landrace (right panel) growing pigs estimated with models 1 and 2, as well as from data corrected for fixed effects (sex and batch) of each test week separately.

day variance for the first eight weeks of the testing period (Fig. 8). Afterwards, the phenotypic test day variance estimated with model 1 fits the estimates from data of single test days corrected for fixed effects better. This is just because in late test weeks the underestimated residual variance of model 1 partly compensates for the overestimated daily variance due to random regression coefficients, which is even higher with model 1 than with model 2 (Figs. 4 and 5). Therefore model 2 is preferred over model 1.

\subsection{Genetic eigenfunctions and eigenvalues}

Any conceivable evolutionary change in a populations mean feed intake curve can be written in terms of a weighted sum of the eigenfunctions. The rate at which a population will evolve from its current mean feed intake curve to some new curve favoured by selection is determined by the eigenvalues associated with eigenfunctions responsible for that change. A large eigenvalue indicates that a change corresponding to that eigenfunction will happen rapidly, while a small eigenvalue indicates that the change will be slow [15].

Eigenfunctions calculated from estimates of genetic (co)variance matrices of random regression coefficients do not differ much between models and are also very similar for the two breeds (Fig. 9). Between 83 and $90 \%$ of the genetic variance for the course of daily feed intake is explained by the first eigenfunction, without change of signs but increasing difference from zero during the testing period. This means that selection in one direction at any time during the testing period will cause a response in the same direction over the whole period, which would be bigger for the last than for the first weeks of the testing period. The second eigenfunction changes signs shortly after nine 

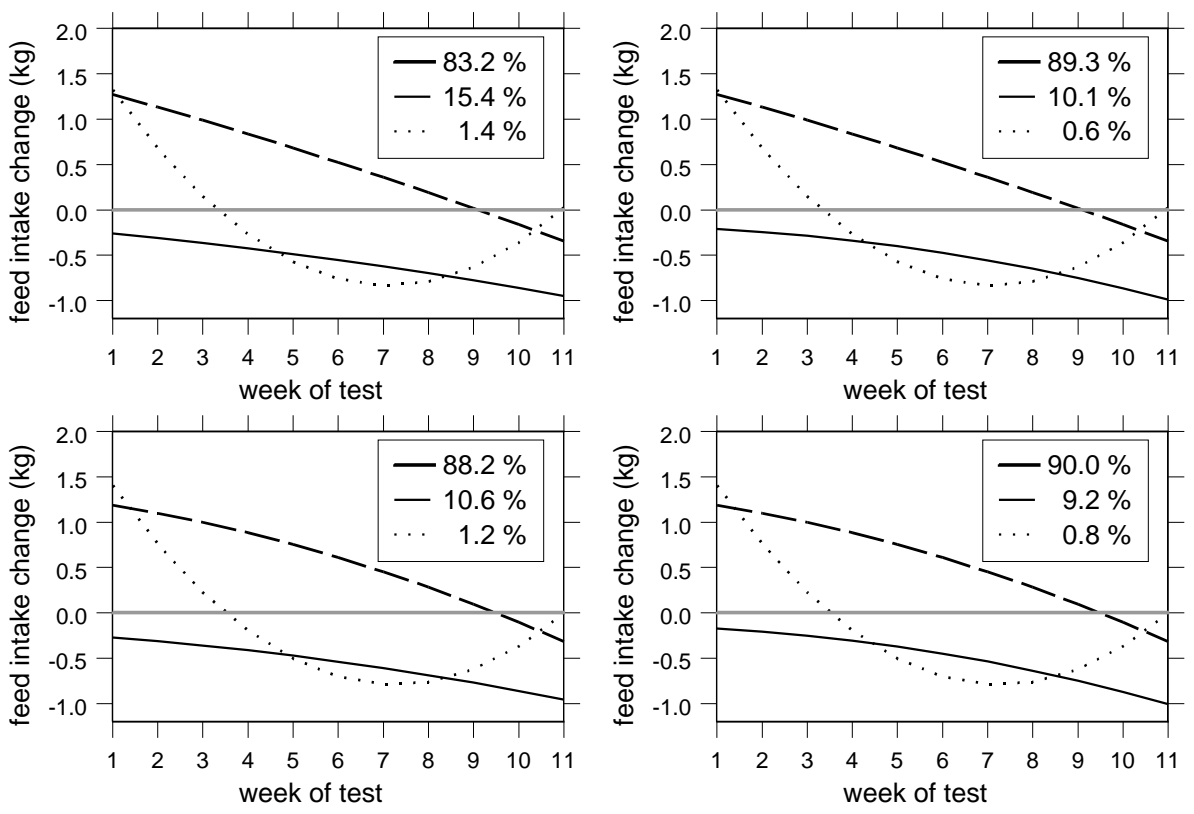

Figure 9. Eigenfunctions for daily feed intake $(\mathrm{kg})$ of Large White (left panels) and French Landrace (right panels) growing pigs for models 1 (upper panels) and 2 (lower panels). Eigenvalues transformed to a percent scale (legend) indicate relative importance of corresponding eigenfunctions.

weeks of the testing period, which is when the fastest growing pigs already reached the desired slaughter weight. Its response to selection would be bigger in the beginning than towards the end of the testing period, while the opposite applies to the first eigenfunction. The third eigenfunction changes signs earlier in the testing period, but explains less than one percent of the variance in feed intake curves, which is negligible. Selection for higher feed intake in the beginning of the testing period, and constant or lower feed intake towards the end, would involve the second (for increasing feed intake in the beginning), as well as the first eigenfunction (for decreasing feed intake towards the end of the testing period). Much more weight would have to be placed on the second eigenfunction, as its associated eigenvalue is much smaller than that of the first eigenfunction. Changing feed intake curves by selection in the desired way thus seems to be difficult, although not impossible.

\section{CONCLUSIONS}

Random regression coefficients provide more information on daily feed intake of growing fattening pigs than a simple mean over the whole testing 
period. The amount of information is comparable to a multivariate analysis of weekly means of feed intake per day, taken over the whole testing period. The advantage of the random regression model is, that fewer parameters (traits) are needed to describe this information. But it is not sure, whether this additional information can be used to improve efficiency of lean growth. Flat posterior distributions of genetic correlations indicate, that information on genetic regression coefficients (especially linear and quadratic) seems to be limited in the data. This may be because the number of animals with records was quite low compared to the high number of levels of genetics effects to be estimated. This lack of information and the complexity of the random regression model seem to be the main reasons for the slow mixing of Gibbs chains of genetic (co)variances. From heritabilities of random regression coefficients of feed intake curves we conclude that changes of the overall level are easier to achieve than changes of slope or inflexion of feed intake curves. Genetic eigenfunctions also reveal that an improvement of feed efficiency by selection on the shape of feed intake curves seems difficult. For a final assessment of possible routes of improvement of efficiency of lean growth by means of selection on feed intake curve parameters, correlations with other traits might be helpful, such as with daily gain, feed conversion ratio and carcass traits. For this, further research is needed.

\section{ACKNOWLEDGEMENTS}

The authors thank Dr. Karin Meyer, Animal Genetics and Breeding Unit, University of New England, Armidale, Australia for the many valuable comments and discussions that helped to improve this article.

\section{REFERENCES}

[1] Andersen S., Pedersen B., Growth and food intake curves for group housed gilts and castrated male pigs, Anim. Sci. 63 (1996) 457-464.

[2] Cole D.J.A., Chadd S.A., Voluntary food intake of growing pigs, in: Forbes J.M., Varley M.A., Laurence T.L.J. (Eds.), The Voluntary Food Intake of Pigs, Occasional Publication of the British Society of Animal Production 13, 1989, pp. 61-70.

[3] De Haer L.C.M., de Vries A.G., Effects of genotype and sex on the feed intake pattern of group housed growing pigs, Livest. Prod. Sci. 36 (1993) 223-232.

[4] De Vries A.G., Kanis E., A growth model to estimate economic values for food intake capacity in pigs, Anim. Prod. 55 (1992) 241-246.

[5] De Vries A.G., Kanis E., Swine breeding goals, short- and long-term considerations, in: Proc. 5th World Congress on Genetics Applied to Livestock Production, 7-12 August 1994, Vol. 17, Guelph, Ontario, 1994, pp. 390-397. 
[6] Eissen J.J., Breeding for feed intake capacity in pigs, Doctoral Thesis, Animal Breeding and Genetics Group, Wageningen University, P.O. Box 338, $6700 \mathrm{AH}$ Wageningen, 2000.

[7] Fowler V.R., Biological advances towards genetic improvement in pigs, in: Proc. 3rd World Congress on Genetics Applied to Livestock Production, 1622 July 1986, Vol. XI, Genetics of Reproduction, Lactation, Growth, Adaptation, Disease, and Parasite Resistance, Lincoln, Nebraska, 1986, pp. 345-354.

[8] Gelman A., Carlin J.B., Stern H.S., Rubin D.B., Bayesian Data Analysis, Chapman \& Hall, London, 1995.

[9] Geman S., Geman D., Stochastic relaxation, Gibbs distributions, and the Bayesian restoration of images, IEEE Transactions on pattern analysis and machine intelligence, PAMI 6 (1984) 721-741.

[10] Geyer C.J., Practical Markov chain Monte Carlo, Stat. Sci. 7 (1992) 473-511.

[11] Hall A.D., Hill W.G., Bampton P.R., Webb A.J., Genetic and phenotypic parameter estimates for feeding pattern and performance test traits in pigs, Anim. Sci. 68 (1999) 43-48.

[12] Hastings W.K., Monte Carlo sampling methods using Markov chains and their applications, Biometrika 57 (1970) 97-109.

[13] Johnson V.E., Studying convergence of Markov chain Monte Carlo algorithms using coupled sample paths, J. Am. Stat. Assoc. 91 (1996) 154-166.

[14] Kanis E., de Vries A.G., Optimization of selection for food intake capacity in pigs, Anim. Prod. 55 (1992) 247-255.

[15] Kirkpatrick M., Lofsvold D., Bulmer M., Analysis of the inheritance, selection and evolution of growth trajectories, Genetics 124 (1990) 979-993.

[16] Labroue F., Aspects génétiques du comportement alimentaire chez le porc en croissance, Thèse de Doctorat, Ensar, Rennes, 1996.

[17] Labroue F., Gueblez R., Sellier P., Genetic parameters of feeding behaviour and performance traits in group-housed Large White and French Landrace pigs, Genet. Sel. Evol. 29 (1997) 451-468.

[18] MathSoft, S-Plus, Version 3.4 for UNIX, Supplement, Data Analysis Products Division, MathSoft, Incorporation, Seattle, Washington, 1996.

[19] Metropolis N., Rosenbluth A.W., Rosenbluth M.N., Teller A.H., Teller W., Equation of state calculations by fast computing machines, J. Chem. Phys. 21 (1953) 1087-1092.

[20] Raftery A.E., Lewis S., How many iterations in the Gibbs sampler?, in: Bernardo J.M., Berger J.O., Dawid A.P., Smith A.F.M. (Eds.), Bayesian Statistics 4, Oxford University Press, Oxford, UK, 1992, pp. 763-773.

[21] Roberts G.O., Markov chain concepts related to sampling algorithms, in: Gilks W.R., Richardson S., Spiegelhalter D.J. (Eds.), Markov chain Monte Carlo in Practice, Chapman \& Hall, London, 1996, pp. 45-57.

[22] Schaeffer L.R., Dekkers J.C.M., Random regression in animal models for testday production in dairy cattle, in: Proc. 5th World Congress on Genetics Applied to Livestock Production, 7-12 August 1994, Vol. 18, Guelph, Ontario, 1994, pp. 443-446.

[23] Sorensen D.A., Andersen S., Gianola D., Korsgaard I., Bayesian inference in Threshold models using Gibbs sampling, Genet. Sel. Evol. 27 (1995) 229-249. 
[24] Stahel W.A., Statistische Datenanalyse, Eine Einführung für Naturwissenschaftler, Vieweg, Braunschweig/Wiesbaden, 1995.

[25] Von Felde A., Roehe R., Looft H., Kalm E., Genetic association between feed intake and feed intake behaviour at different stages of growth of group-housed boars, Livest. Prod. Sci. 47 (1996) 11-22.

[26] Webb A.J., Genetic control of growth, composition, appetite and feed utilisation: non-ruminants, Options for genetic change, in: Proc. 3rd World Congress on Genetics Applied to Livestock Production, 16-22 July 1986, Vol. XI, Genetics of Reproduction, Lactation, Growth, Adaptation, Disease, and Parasite Resistance, Lincoln, Nebraska, 1986, pp. 337-344.

[27] Webb A.J., Future challenges in pig genetics, Anim. Breed. Abstr. 63 (1995) 731-736.

To access this journal online: www.edpsciences.org 